\title{
Investigating Distributed Sensor/Point Actuator Testbeds by Using Differential Laser Doppler Interferometer
}

\author{
C. K. Lee, ${ }^{*}$ W. H. Hsiao, ${ }^{\dagger}$ H. C. Shih, ${ }^{\dagger}$ and C. T. $\operatorname{Lin}^{\dagger}$ \\ National Taiwan University, Taipei 10764, Taiwan, Republic of China \\ and \\ Thomas C. T. Teng ${ }^{\ddagger}$ and James G. Y. Wu ${ }^{\S}$ \\ AHEAD Optoelectronics, Inc., Taipei 235, Taiwan, Republic of China
}

\begin{abstract}
The design principles, optical configurations, and capabilities of a newly developed advanced vibrometer/interferometer device called AVID are presented. The fundamental principles of this metrology instrument that enables it to have a de to $20-\mathrm{MHz}$ bandwidth and nanometer resolution are also detailed. The applicability of using laminated one-dimensional piezoelectric plates as testbeds to study some of the fundamental characteristics of piezoelectric actuators and sensors is also examined. Taking advantages of the linear and angular displacement/velocity measurement modes of the vibrometer/interferometer, the performance of distributed piezoelectric sensors, which can measure bending angle and bending displacement at any point of the testbed, is characterized. In addition, the corresponding piezoelectric actuator that can exert bending moment on the testbed is also evaluated. The hysteresis effect and feedback vibration control potential of both piezoelectric polymer polyvinylidene fluoride $\left(\mathrm{PVF}_{2}\right)$ and piezoelectric ceramic lead, zirconate, and titarate $(\mathrm{PZT})$ are investigated by using a bimorph configuration testbed. Experimental results show that $\mathbf{P V F}_{2}$ acts more linearly than PZT during sensing. Furthermore, the influence of the driving sources on the piezoelectric actuators can be verified experimentally. It can be shown that driving PZT by using a current source will have a less severe hysteresis effect than driving it with a traditional voltage source.
\end{abstract}

\section{Introduction}

$\mathbf{T}$ HERE are multifold objectives in this paper. The first is to report the design thinking, optical configurations, and capabilities of the newly developed advanced vibrometer/interferometer device named AVID. The second is to examine the applicability of using a laminated one-dimensional piezoelectric plate as a testbed to study some of the fundamental characteristics of piezoelectric actuators and sensors. The third is to use AVID and the testbed to examine ways of reducing the hysteresis effect of piezoelectric sensors/actuators.

Since the Curie brothers first discovered the piezoelectric effect in 1880 , more than 100 different piezoelectric materials have become available. In fact, piezoelectric materials are probably the most popular materials to be integrated into electric mechanical systems. Generally speaking, there are two known kinds of piezoelectric materials. The first kind includes ceramics or crystals and is characterized by being brittle in nature. One of the most commonly used materials in this category is PZT (lead, zirconate, and titanate). Piezopolymers, the second type of piezoelectric material, are characterized as being flexible and soft when compared to PZT. Polyvinylidene fluoride $\left(\mathrm{PVF}_{2}\right)$, discovered in 1969 , is the most often adopted pieozopolymer as it possesses one of the strongest piezoelectricproperties within polymeric materials. It is well known that all piezoelectric materials possess a nonlinear relationship between an electric field and stress called the hysteresis effect. The hysteresis effect is typically ignored in practical applications. Certainly, this assumption becomes the very reason to place blame when inaccuracy or instabilityin the control loop arises. To meet the

Received 6 February 1998; presented as Paper 98-2077 at the AIAA/ASME/ASCE/AHS/ASC 39th Structures, Structural Dynamics, and Materials Conference, Long Beach, CA, 20-23 April 1998; revision received 10 August 2000; accepted for publication 21 August 2000. Copyright (C) 2000 by the American Institute of Aeronautics and Astronautics, Inc. All rights reserved.

*Professor, Institute of Applied Mechanics; cklee@ mems.iam.ntu.edu.tw. Senior Member AIAA.

Assistant Researcher, Institute of Applied Mechanics.

†ngineer

$\S$ Manager. high-precisionneeds of high-performancemechatronic systems, the hysteresis effect should be part of the design process at the very beginning. Methodologies such as examining the possibility of adopting a more linear piezoelectric sensing material to tailor or linearize the performance of a more powerful piezoelectric actuator material deserves more attention. In addition, as piezoelectric materials possess high-output impedance, it is expected that their performance will be highly influenced by the circuitry connected to it. Such sensing performance has been investigated previously and has led to the development of piezoelectric strain rate gauges. ${ }^{1}$ The relationship between the hysteresis effect of PZT and the driving circuit will be examined in detail in this paper.

To facilitate the control to structure interaction evaluation and to satisfy the metrology needs of today's ultra-high-performance mechanic/mechatronic systems, an instrument that has wide bandwidth (up to megahertz) and high resolution (to nanometers) is highly desirable. To meet this requirement, an innovative advanced vibrometer/interferometer device called AVID was developed. ${ }^{2,3}$ AVID is essentially a differential laser Doppler interferometer, which can use either a $\mathrm{He}-\mathrm{Ne}$ laser or a wave-front-corrected diode laser as the light source. The circular polarization interferometer configuration, which removes the acoustic optical modulator (AOM) such as in traditional vibrometers, allows AVID to be miniaturized and to circumvent the electromagnetic interference (EMI) effect induced by the high-frequency driving signal of the AOM. Furthermore, a new signal-decoding algorithm was developed to compensate the less than ideal quadrature signals encountered in practical applications, which reduces the noise efficiently in order to achieve nanometer measurement accuracy. A new circuitry was designed to provide AVID from a range of dc to $20-\mathrm{MHz}$ bandwidth, which is approximately 60 times wider than typical vibrometers. In addition, both linear and angular velocity/displacements can be directly derived by using either AVID's dual-beam or singlebeam configuration to ease the mechanical system performance evaluations.

\section{Theory}

All of the theories related to piezoelectric laminate testbeds and AVID are discussed in detail here. 


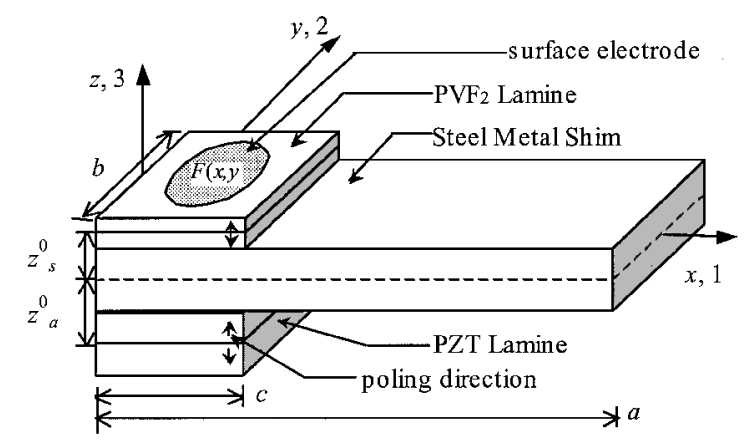

Fig. 1a Basic configuration of the piezoelectric laminate of the twolayered $\mathrm{PVF}_{2}$ design.

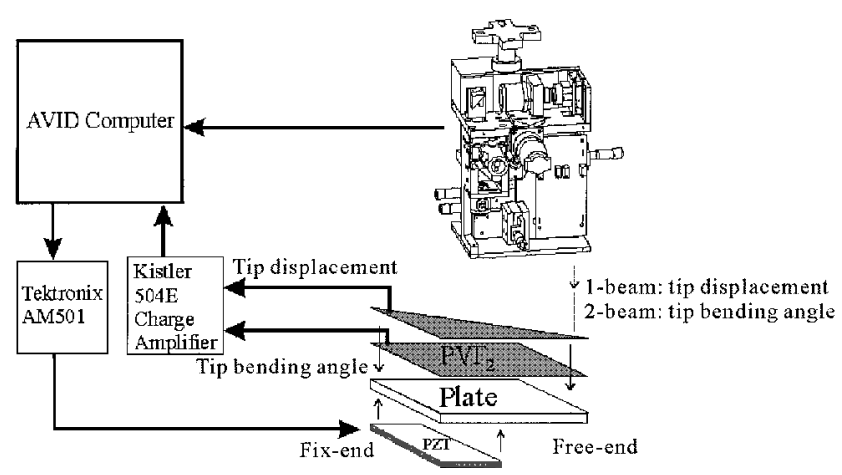

Fig. 1b Basic configuration of the piezoelectric laminate of the fourlayered $\mathrm{PVF}_{2}$ design.

\section{Governing Equations of the Testbed}

The hysteresis effect of piezoelectric material was examined in a bimorph configuration as a result of the case in generating large strains on piezoelectric materials. A thin, one-dimensional cantilever laminated plate measuring $14 \mathrm{~cm}$ long and $2 \mathrm{~cm}$ wide (Fig. 1) was chosen as the basic testbed configuration where all theory and experimental work were based.

The first testbed configuration (Fig. 1a) was laminated by using a $180-\mu \mathrm{m}$ (7-mils)-thick stainless-steel shim as the center layer, two layers of $110-\mu \mathrm{m}$-thick rectangular-shapedPVF 2 and two layers of the same shaped 250- $\mu \mathrm{m}$ PZT bounded to the top and the bottom surfaces of the center layer as the piezoelectric laminae. The second testbed configuration (Fig. 1b), shown with the AVID system located above the free end of the tip, has two layers of a rectangular $\mathrm{PVF}_{2}$ covering the whole surface, two layers of a triangular-shaped $\mathrm{PVF}_{2}$, and two rectangular-shapedPZT of the same size as that of the first testbed configuration. The double-layered design serves to shield the signals from EMI noise and to increase the signal/noise ratio. ${ }^{4,5}$ The poling axis is defined as the $z$ axis, and the stretching (rolling) direction is denoted as the $x$ axis. To simplify the problem, a zero skew angle ${ }^{5,6}$ was chosen to avoid sensing torsion and shear.

\section{Lamina Constitutive Relationship}

From the Kirchhoff hypothesis a one-dimensional thin plate can be considered in the plane-stress state. This understanding leads to the piezoelectric lamina constitutive relationship, which possesses the form shown next when written in a list of Electrical and Electronics Engineers compact matrix notation ${ }^{4,7}$ as follows:

$$
\begin{gathered}
\left.\left[\begin{array}{c}
T_{1} \\
T_{2} \\
T_{6}
\end{array}\right]=c\right]\left[\begin{array}{l}
S_{1} \\
S_{2} \\
S_{6}
\end{array}\right]-E_{3}\left[\begin{array}{c}
e_{31} \\
e_{32} \\
0
\end{array}\right] \\
D_{3}=\varepsilon_{33} E_{3}+d_{31} T_{1}+d_{32} T_{2}
\end{gathered}
$$

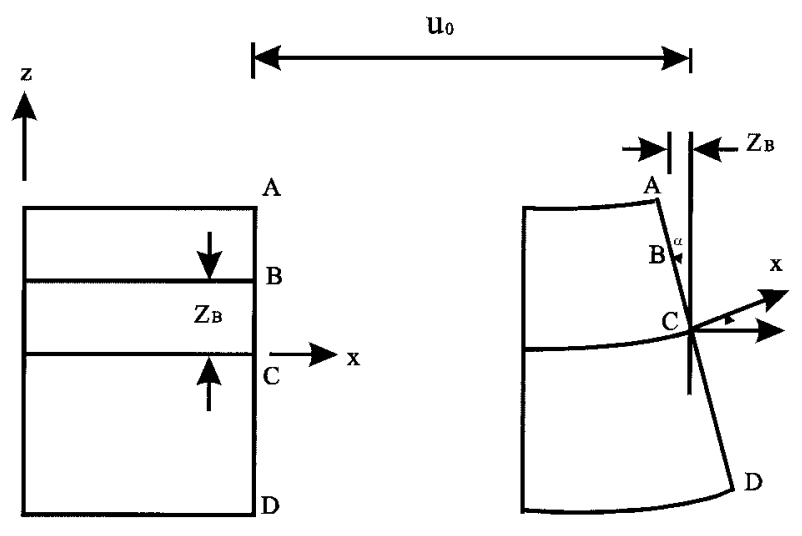

a)

b)

Fig. 2 The $x-z$ plane: a) initial and b) deformed.

where

$$
c]=\left[\begin{array}{ccc}
Y /\left(1-v^{2}\right) & v Y /\left(1-v^{2}\right) & 0 \\
v Y /\left(1-v^{2}\right) & Y /\left(1-v^{2}\right) & 0 \\
0 & 0 & Y / 2(1+v)
\end{array}\right]
$$

the vectors $\boldsymbol{T}, \boldsymbol{S}, \boldsymbol{D}$, and $\boldsymbol{E}$ represent the stress, strain, electric displacement, and electric field, respectively, and $\varepsilon$ is the permittivity constant of the piezoelectric lamina. In addition, even though $c$ ] represents the elastic stiffness matrix of the piezoelectric lamina it is assumed to have the form of the elastic stiffness matrix of an isotropic material in order to simplify the problem. This assumption can be justified by considering that $\mathrm{PVF}_{2}$ is significantly softer than that of the stainless-steel shim and that PZT is actually transverse isotropic. The constants $\boldsymbol{e}$ and $\boldsymbol{d}$ are the piezoelectric stress/charge and strain/charge constants of the lamina and are related by

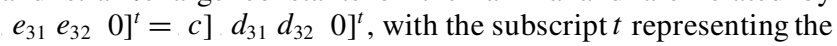
vector transposition.

\section{Strain-Displacement Relationship}

Considering point $c$ as the midplane of the $x-z$ plane (Fig. 2), line $\mathrm{AD}$ as normal to the midplane, and line ACD remaining straight after the deformation, the deformation of point $\mathrm{B}$ can be shown to be $u_{B}=u_{0}-z_{B} \alpha$ with $\alpha=\partial w / \partial x$ and $w$ as the transverse displacement. The displacement $u$ in the $x$ direction and $v$ in the $y$ direction are thus $u=u_{0}-z \partial w / \partial x$ and $v=v_{0}-z \partial w / \partial y$. The linear elasticity theory leads further to $S_{1}=\partial u / \partial x, S_{2}=\partial v / \partial y, S_{6}=$ $\partial u / \partial x+\partial v / \partial y$ (Ref. 8). Combining all of the preceding relationships yields the strain-displacementrelationship

$$
\begin{aligned}
{\left[\begin{array}{l}
S_{1} \\
S_{2} \\
S_{6}
\end{array}\right]=} & {\left[\begin{array}{l}
S_{1}^{0} \\
S_{2}^{0} \\
S_{6}^{0}
\end{array}\right]+z\left[\begin{array}{l}
k_{1} \\
k_{2} \\
k_{6}
\end{array}\right]=\left[\begin{array}{c}
\partial u_{0} / \partial x \\
\partial v_{0} / \partial y \\
\partial u_{0} / \partial x+\partial v_{0} / \partial y
\end{array}\right] } \\
+z & {\left[\begin{array}{c}
-\partial^{2} w / \partial x^{2} \\
-\partial^{2} w / \partial y^{2} \\
-2 \partial^{2} w / \partial x \partial y
\end{array}\right] }
\end{aligned}
$$

where $k_{1}$ and $k_{2}$ are curvatures and $k_{6}$ represents the twisting angle.

\section{Sensor Equation}

As was just mentioned, piezoelectric materials have high impedance in sensing, and as such the signals measured are strongly influenced by the interfacing circuitry used to couple the signals to the measuring instrument. For example, if a charge amplifier is used the closed-circuit charge signal, i.e., $E_{3}=0$, will lead to a strain or displacement measurement. ${ }^{1,5}$ More specifically, the charge signal $q(t)$ enclosed by the effectivesurface electrode $A$ along the direction of the thickness can be calculated by using Gauss's law:

$$
q(t)=\iint_{A} D_{3} \mathrm{~d} x \mathrm{~d} y
$$


where $D_{3}$ is the electric displacement. As the transverse displacement $w$ is a function of the $x$ only, combining Eqs. (1-4) yields the charge sensor equation

$$
q(t)=-z_{s}^{0} \iint_{A} e_{31} \frac{\partial^{2} w}{\partial x^{2}} \mathrm{~d} x \mathrm{~d} y
$$

where $z_{s}^{0}$ is the moment arm of the piezoelectric sensor. ${ }^{1,5}$

On the other hand, if a current amplifier is used to couple the signal a strain rate or velocity measurement will result. Following similar procedures, the current sensor equation becomes

$$
i(t)=\frac{\mathrm{d} q(t)}{\mathrm{d} t}=-z_{s}^{0} \iint_{A} e_{31} \frac{\partial^{3} w}{\partial x^{2} \partial t} \mathrm{~d} x \mathrm{~d} y
$$

If a voltage amplifier is connected to the piezoelectric sensor, the relationship $\boldsymbol{e}_{\mathrm{ip}}=\varepsilon_{\mathrm{ij}} \boldsymbol{h}_{\mathrm{jp}}$ between the piezoelectric constants $\boldsymbol{h}_{\mathrm{jp}}$ and piezoelectric strain/stress constants $\boldsymbol{e}_{\text {ip }}$ applies. The voltage sensor equation can then be written as

$$
v(t)=\frac{q(t)}{C_{a}}=-z_{s}^{0} \frac{h_{s}}{A} \iint_{A} h_{31} \frac{\partial^{2} w}{\partial x^{2}} \mathrm{~d} x \mathrm{~d} y
$$

where $C_{a}=\varepsilon_{33} A / h_{s}$ is the capacitance and $h_{s}$ presents the thickness of the piezoelectric lamina. One factor that should be noted in choosing the interfacing circuitry is the temperature stability and linearity of piezoelectricconstants $\boldsymbol{h}_{\mathrm{jp}}$ and piezoelectricstrain/stress constants $\boldsymbol{e}_{\text {ip }}$. For example, although the piezoelectric constants $\boldsymbol{h}_{\mathrm{jp}}$ of $\mathrm{PVF}_{2}$ are more temperature stable than the piezoelectric strain/stress constants $\boldsymbol{e}_{\text {ip }}$ the opposite is true for PZT. Because of the reciprocal relationship that exists between piezoelectric sensors and actuators, ${ }^{1,6}$ it is expected that driving a piezoelectric actuator by controlling an externally applied voltage will be quite different from driving it by setting the externally applied charge. More specifically, as $\boldsymbol{T}_{1} \sim \boldsymbol{e}_{31} \boldsymbol{E}_{3} \sim \boldsymbol{h}_{31} \boldsymbol{D}_{3}$ the linearity of $\boldsymbol{e}_{31}$ vs $\boldsymbol{h}_{31}$ will dictate the linearity of the actuator. It will be shown that driving the PZT by controlling the charge is more linear than driving the PZT by exerting voltage.

The same-sized rectangular PZT and $\mathrm{PVF}_{2}$ were adopted in the first testbed configuration, that is, the effective surface electrode pattern is

$$
F=H(x)-H(x-c)] H(y-b / 2)-H(y+b / 2)]
$$

where $H(x)$ is the Heaviside step function and $c$ is the length of the $\mathrm{PZT}$ and $\mathrm{PVF}_{2}$. Because the derivative of the Heaviside step function is the Dirac delta function $\delta(x)$, the sensing terms appearing to the right of Eqs. (5-7) become $\mathrm{b}^{1,6}$

$$
\left.\frac{\partial^{2} F}{\partial x^{2}}=\delta^{\prime}(x)-\delta^{\prime}(x-c)\right]\left[H\left(y-\frac{b}{2}\right)-H\left(y+\frac{b}{2}\right)\right]
$$

For the two testbed configurations the transverse displacement $w$ at $x=0$ is zero, and the preceding form indicates that a rectangularshaped piezoelectric lamina will act like a sensor that will sense bending angle or bending angular velocity at $x=c$ depending on the interfacing circuit used. Similarly, the triangular-shaped $\mathrm{PVF}_{2}$ in the second test configuration can be shown to measure transverse displacement $w(x=c)$ (Refs. 9 and 10).

\section{Actuator Equation}

The equation of motion of the testbed, i.e., the actuator equation, can be obtained by introducing Eq. (1) into the moment equilibrium equation of the classical laminated plate theory., ${ }^{4,9,11}$ Adopting the Kirchhoff hypothesis and assuming that the laminate possesses midplane symmetry, the actuator equation becomes

$$
D_{11} \frac{\partial^{4} w}{\partial x^{4}}+\rho h \frac{\partial^{2} w}{\partial t^{2}}=-2 z_{a}^{0} h_{a} e_{31} G(t) \frac{\mathrm{d}^{2} F(x)}{\mathrm{d} x^{2}}
$$

where $D_{11}, \rho$, and $h$ are flexural rigidity, density, and thickness of the piezoelectric laminate; $h_{a}$ is the single-layer thickness of PZT actuator; $z_{a}^{0}$ is the moment arm of the double-layerPZT lamina; $G(t)$ represents the input electric field; and $F(x)$ describes the effective surface electrode. ${ }^{4,6,8,11}$ Even though the piezoelectric lamina may not be transverse isotropic, both $D_{11}$ and $\rho h$ are assumed to be transverse isotropic by neglecting the contribution of the piezoelectric laminas in order to reveal the underlying physical meanings without indulging the whole analysis in the mathematical model used. In other words, $D_{11}$ is assumed to be $\left.\left(h_{s}^{3} / 12\right) Y_{s} /\left(1-v_{s}^{2}\right)\right]$, and $\rho h$ are treated as constants with the subscript $s$ designating the stainless-steel shim.

As was shown in Eq. (9), the forcing term appearing to the left of Eq. (10) indicates that the rectangular-shaped piezoelectric lamina of the first testbed configuration acts like an actuator that can exert a concentrated point moment at $x=c$ (Refs. 9 and 10). In other words, even though this type of PZT actuator is distributed in nature it is in fact a point actuator, which means it can be used to mimic the action of a traditional point actuator. Similarly, the triangularshaped PZT actuator used in the second testbed configuration will exert a concentrated point force at $x=c$ (Refs. 9 and 10), which in Fig. $1 b$ is at the tip of the free end.

\section{Operating Principles of AVID}

The schematics of the three built-in optical configuration of AVID are shown Figs. 3a-3c. The MEMS Laboratory of National Taiwan

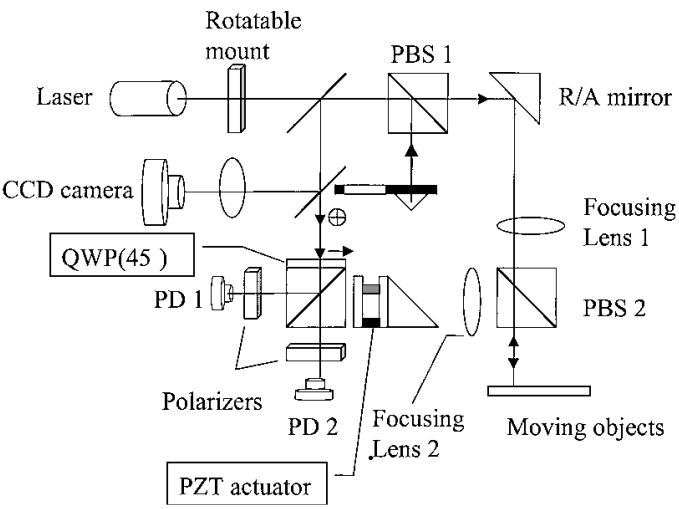

a) Single-beam configuration to measure absolute displacement/velocity

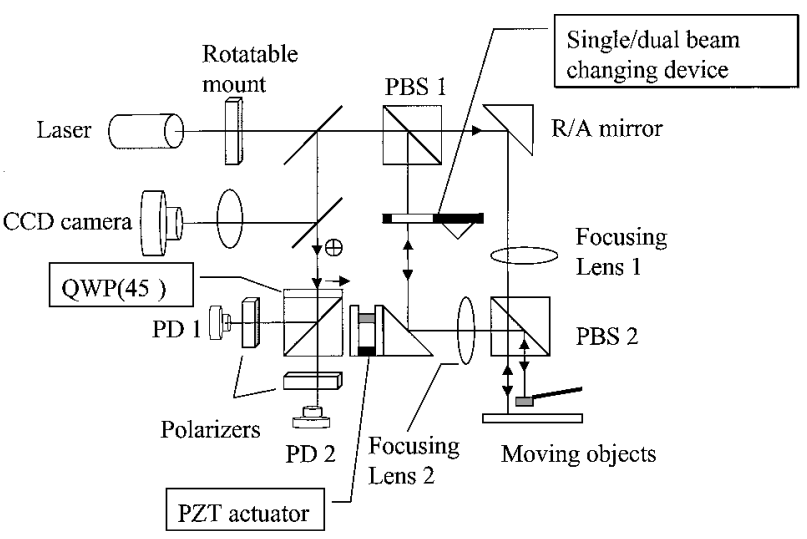

b) Dual-beam configuration to measure relative displacement/velocity, i.e., angle or angular velocity

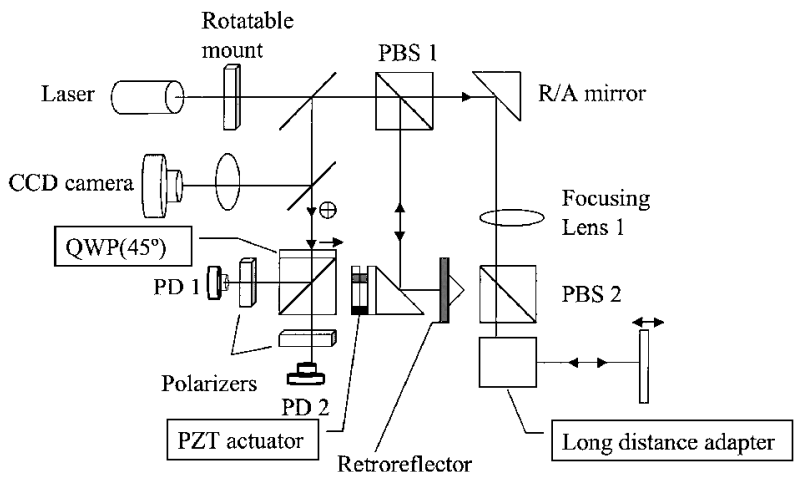

c) Long working interferometer mode to measure displacement/velocity of long distance traveling objects

Fig. 3 Built-in optical configurations of AVID. 


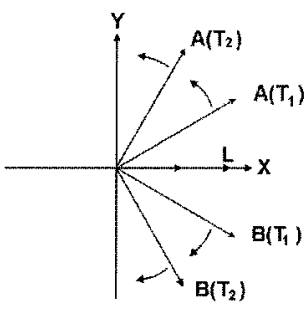

No Relative Movement Net $L=0$

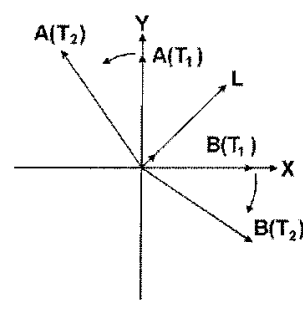

The $\lambda / 4$ Relative Movement Net $L=\lambda / 4$
Fig. 4 Graphic representation of a circular polarization interferometer.

University and AHEAD Optoelectronics, Inc., ${ }^{12}$ jointly developed AVID over the past six years. Its underlying principles are based on the Doppler effect and laser interferometry.

After light beam 1 passes through a right-angle mirror and a polarizationbeamsplitterPBS2, it is focused onto a point on the testbed by a doublet lens L1. The single/dual-beam changing mechanism can be used to transform AVID between a single-beamconfiguration (Fig. 3a) and a dual-beam(Fig. 3b) configuration.In the single-beam configuration light beam 2 is reflected internally by a corner cube to serve as the reference light. Operating AVID in this mode provides us with an opportunity to measure the displacement or velocity at any point on the testbed. When the system is operated in the dualbeam configuration, the outgoing light and returning object beams are diametrically incident on different locations of focusing lenses L1 and L2. The two returning light beams remain linearly polarized and orthogonal with respect to each other after being recombined at the polarization beam splitter PBS1. One thing that should be noted is that even though the polarization states of the two returning light beams can change in some measurement surfaces both PBS1 and PBS2 will serve as the gateway to ensure that the two returning beams retain their proper polarization states.

As was just mentioned, a circularpolarizationinterferometryconfiguration was adopted to remove the directional ambiguity. $2,3,13$ One right circularly polarized light beam and one left circularly polarized light beam are produced after the two returning light beams travel through the quarter waveplate QW(45) whose fast axis is oriented along a 45-deg angle. The vector sum of these two circularly polarized light beams is also a linearly polarized light beam but with an inclination angle relative to the horizontal axis varying according to the motion of the object (Fig. 4). This resultant, a linearly polarized light beam, is then divided into two light beams by a nonpolarizationbeam splitter. The two polarizationbeam splitters PBS3 and PBS4, whose transmission axes are 45 deg apart, are utilized to create the 90-deg phase difference needed to create a quadrature signal at photodiodes PD1-PD4. Velocity and displacement signals can thus be easily decoded. More specifically, the returning beams $E_{1}$ and $E_{2}$ are

$$
\begin{gathered}
\left.E_{1}=\left[\begin{array}{l}
1 \\
0
\end{array}\right] \exp i 2 \pi\left(f_{l}+f_{d 1}\right) t\right] \\
\left.E_{2}=\left[\begin{array}{l}
0 \\
1
\end{array}\right] \exp i 2 \pi\left(f_{l}+f_{d 2}\right) t+\varphi\right]
\end{gathered}
$$

where $f_{l}$ is frequency of the incident laser beam and $f_{d 1}$ and $f_{d 2}$ are the Doppler shift generated from the specimen motion. Considering the case that the amplitudes of the two light beams are identical, the light beam generated by combining the two returning object beams after the quarter waveplate becomes ${ }^{2,3,13}$

$\left.E=\left\{\left[\begin{array}{l}1 \\ i\end{array}\right] \exp \left(i 2 \pi f_{d 1} t\right)+\left[\begin{array}{l}i \\ 1\end{array}\right] \exp i 2 \pi\left(f_{d 2} t+\phi\right)\right]\right\} \exp \left(i 2 \pi f_{1} t\right)$

This light beam is the vector sum of the two circularly polarized light beams and can be viewed as a linearly polarized light with its polarization axis located at $\left.2 \pi\left(f_{d 1}-f_{d 2}\right) t+\phi\right] / 2$ (Fig. 4).

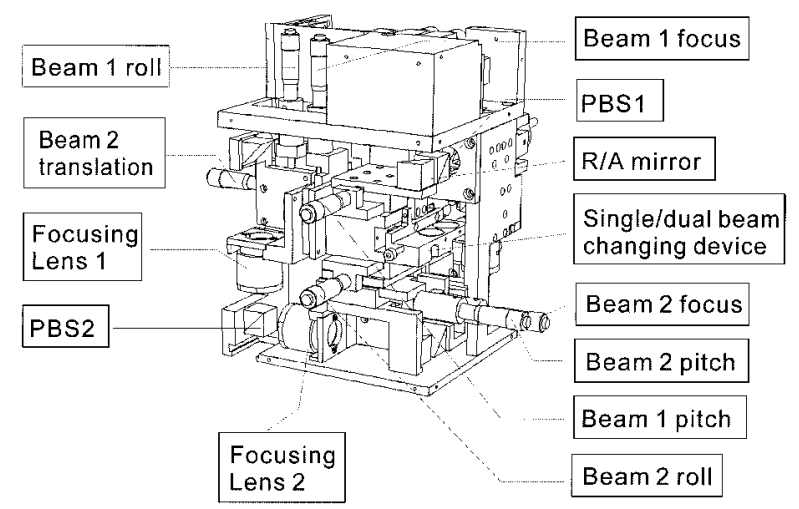

Fig. 5 Built-in mechanical stages of AVID to simplify testing points assignment and alignment.

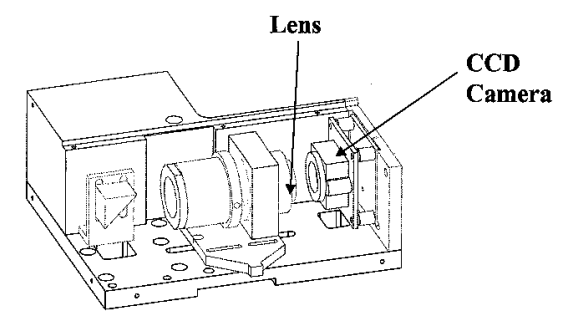

Fig. 6 Built-in microscope of AVID to facilitate optical alignment and sample imaging.

More specifically, the recombined light beam $\boldsymbol{E}$ becomes a linearly polarized light beam with its polarizationaxis rotating through 360 deg whenever the optical path difference between the two light beams reaches two wavelengths. Because the spatial axes of the two polarizing beam splitters PBS3 and PBS4 are 45 deg apart, the quadrature signals detected from the photodiodes can be expressed as follows:

Sine-signal:

$$
\left.I_{1} \propto 1+\sin 2 \pi\left(f_{d 1}-f_{d 2}\right) t+\phi\right]
$$

Cosine-signal:

$$
\left.I_{2} \propto 1+\cos 2 \pi\left(f_{d 1}-f_{d 2}\right) t+\phi\right]
$$

Performing standard quadrature signal detection to the preceding two signals leads to relative displacement between the two object surfaces. Mixing these two signals with an externally generated sinusoidal $\sin \left(2 \pi f_{c} t\right)$ and its 90 -deg phase-shifted signal $\cos \left(2 \pi f_{c} t\right)$ yields an output signal of $\left.\sin 2 \pi\left(f_{c}-f_{d}\right) t\right]$, where $f_{d}=f_{d 1}-f_{d 2}$. Sending the $\left.\sin 2 \pi\left(f_{c}-f_{d}\right) t\right]$ signal into a frequency-to-voltage converter yields a velocity signal. Using these quadrature detection algorithms, AVID is equipped with the capabilities of measuring both velocity and displacement. Other features include built-in stages (Fig. 5), a microscope (Fig. 6) to facilitate optical alignment, and a testbed metrology points allocation. ${ }^{2,3,13}$ All of these features are packed into a small 10 by 12 by $15.5 \mathrm{~cm}$ volume (Fig. 7) to ease its use.

\section{Experiments}

The hysteresis effect related to the sensing and actuating behavior piezoelectric materials were evaluated. The objective related to sensing was to evaluate the linearity of $\mathrm{PVF}_{2}$ and PZT in order to identify the possibility of using a more linear piezoelectric material to tailor the performance of a more powerful piezoelectric actuator. The actuating approach objective was to try and identify a more linear driving approach in order to minimize the hysteresis effect of piezoelectric actuators.

\section{Testbed Evaluations by Using AVID}

The $\mathrm{PVF}_{2}$ sensors, which are produced by Measurement Specialties, Inc., in thickness of $110 \mu \mathrm{m}$ shown in Fig. 1b, were chosen to cross-calibrate piezoelectric sensors with AVID. As was 


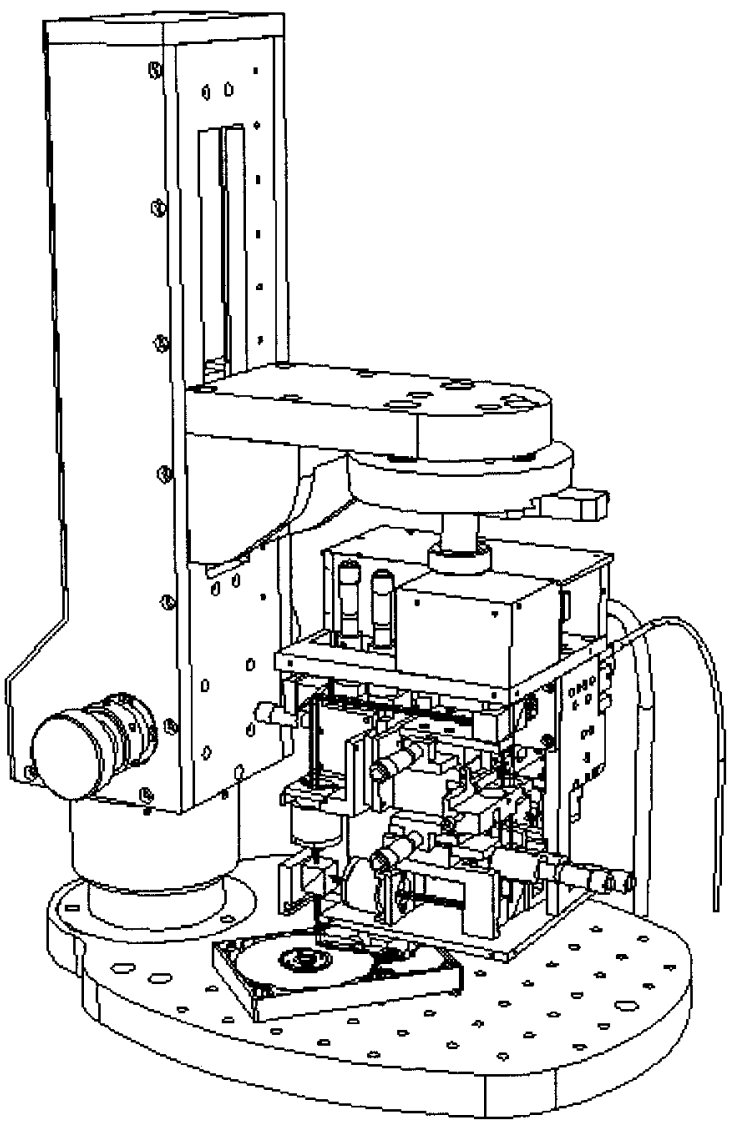

Fig. 7 Exterior view of AVID optical metrology head.

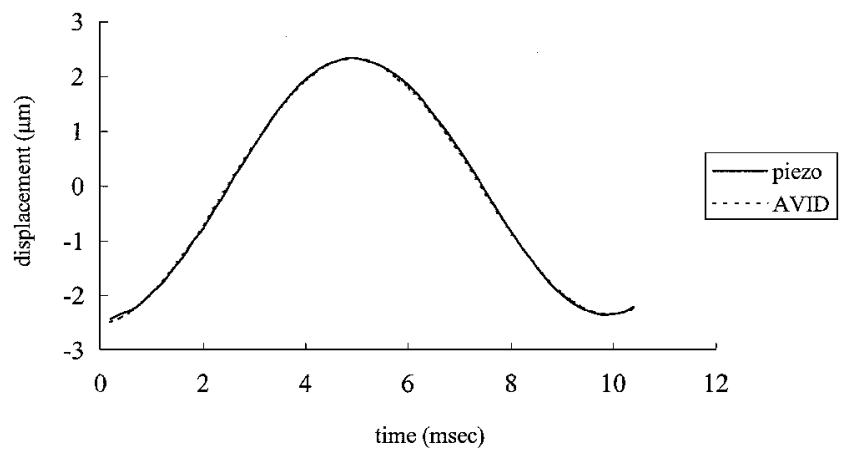

Fig. 8 Tip displacement of a cantilever beam measured by using a) the single-beam AVID configuration and $b$ ) the charge signal of $P F_{2}$ sensors where the transimpedance gain of the charge amplifier was set at $107 \mathrm{~V} / \mathrm{C}$ and the sampling rate was chosen to be $50 \mathrm{KHz}$. The phase difference between the two signals is less than $0.77 \mathrm{deg}$.

just described, the uniform sensor can measure the tip bending angle, whereas the triangle-shaped $\mathrm{PVF}_{2}$ sensor can measure the tip displacement. Piezo Systems, Inc.'s PZT bending element T215A4-303, which is produced by PZT5A, was pasted on the opposite surface of the cantilever beam to excite the testbed. The dimensions of the cantilever beam made by stainless steel is $14 \times 2.2 \mathrm{~cm} \times 200 \mu \mathrm{m}$. A Kistler 504E dual-mode amplifier ${ }^{14}$ was used as the charge amplifier for piezoelectric sensors, and a Tektronix AM501 amplifier ${ }^{15}$ was used to amplify the driving signal command issued from a Pentium-based PC. The experimental results obtained are shown in Figs. 8 and 9. In comparison, a dual-beam AVID configuration (Fig. 3b) measures tip bending angle, whereas a single-beam AVID configuration (Fig. 3a) measures tip displacement. Comparing the results obtained from the two $\mathrm{PVF}_{2}$ sensors vs that from AVID creates an excellent cross-calibration platform. It is clearly seen from the experimental results shown in Figs. 8 and 9 that the signals obtained from $\mathrm{PVF}_{2}$ and AVID agree well, which veri-

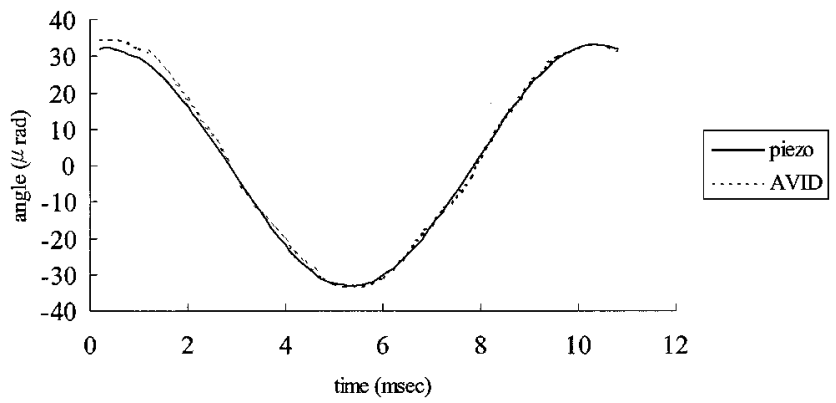

Fig. 9 Tip displacement of a cantilever beam measured by using a) the dual-beam AVID configuration and $b$ ) the charge signal of $P F_{2}$ sensors where the transimpedance gain of the charge amplifier was set as $107 \mathrm{~V} / \mathrm{A}$ and the sampling rate was chosen to be $50 \mathrm{KHz}$. The phase difference of the two signals is less than $0.56 \mathrm{deg}$.

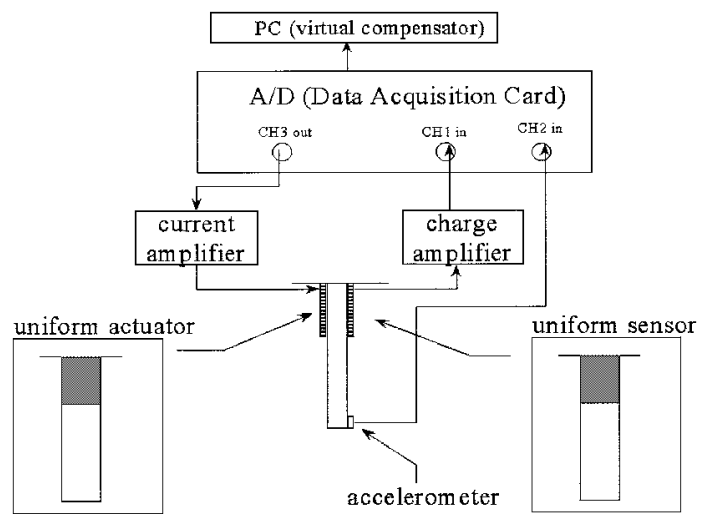

Fig. 10 Experimental setup for measuring the linearity of PZT and $\mathbf{P V F}_{2}$ in sensors.

fies the validity of the theoretical predictions and the effectiveness of the testbed configurations.

Plotting the experimental results of Figs. 8 and 9 clearly reveals the hysteresis effect of $\mathrm{PVF}_{2}$ sensors. To further quantify the linearity of $\mathrm{PVF}_{2}$ and that of the PZT, the first testbed configuration was used for further evaluations.

\section{Linearity of PZT and $\mathrm{PVF}_{2}$ Sensors}

As was just mentioned, the two piezoelectric sensors and actuators were designed and fabricated in the experiments related to the first testbed configuration (Figs. 1a and 10). According to the theory developed by Martin in 1977 (Ref. 16), the transfer functions of the collocated and compatible actuators/sensors pairs for an undamped elastic body will have alternating poles and zeros on the imaginary axis, which ensures the control system stability. The experiment with the same size $\mathrm{PVF}_{2}$ sensor and PZT actuator forms a co-located sensor/actuator pair, which can be used for future control-loop evaluations.

In addition to AVID, an Endevco PC22 miniature accelerometer ${ }^{17}$ was also attached to the free end of the cantilever plate to measure the harmonic oscillations of the cantilever plate. Signals generated from the accelerometer were connected to a charge amplifier to convert it into a voltage signal in order to be recorded for further processing. The PZT and $\mathrm{PVF}_{2}$ sensor signals were also connected to their respective current amplifiers. All of the signals were digitized by an A/D data acquisition card ${ }^{18}$ installed within a Pentium-based PC running under a LabVIEW environment. ${ }^{19}$ A graphic user interface instrument control and data analysis program was developed to execute the experiments.

A small plastic stick was used to excite the laminated plate with an initial impulse. The impulse induced free vibration on the lightly damped plate, which in turn generated sensor signals. The signals generated from AVID, the accelerometer, and the uniform rectangular-shapedPVF 2 and PZT sensors were all recorded by using a PC-based experimental setup as shown in Fig. 10. 


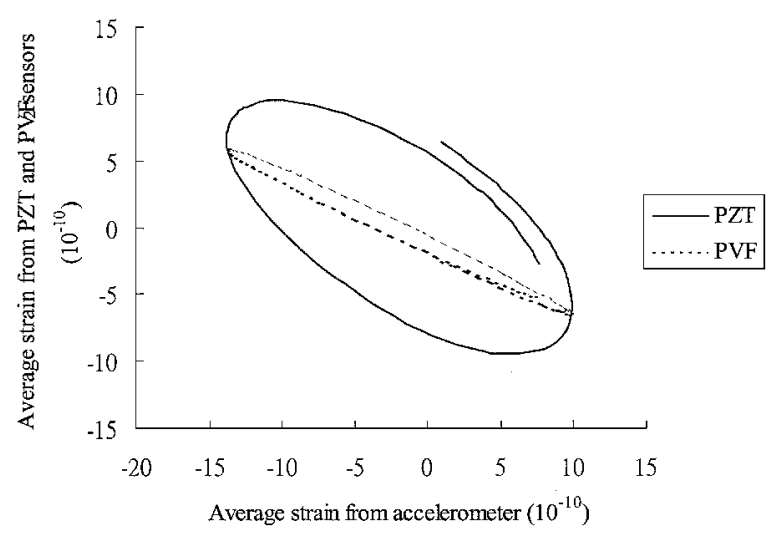

Fig. 11 Hysteresis curve of $\mathrm{PVF}_{2}$ and PZT sensors.

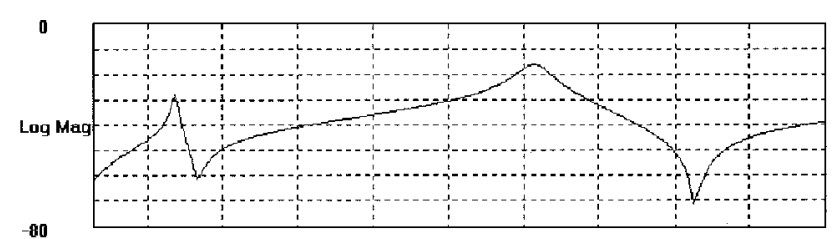

200

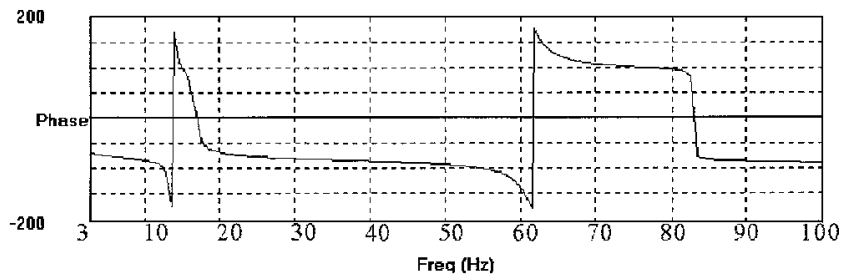

Fig. 12 Transfer function of the second testbed configuration.

For data analysis the signal generated by either AVID or the accelerometer was used to recover the average strain signal, which appeared at the sensor location. The initial deformation induced by the plastic stick was found to excite mostly the first mode on the cantilever laminated plate. For piezoelectric sensors Eq. (6) was adopted to calculate the average strain as current amplifiers were used as the interfacing circuit. Assuming that the strain data measured by AVID or the accelerometer were precise, the hysteresis effect of PZT and $\mathrm{PVF}_{2}$ sensors can then be compared by plotting the strain-strain graph for each piezoelectric lamina (Fig. 11). It is clear from Fig. 11 that $\mathrm{PVF}_{2}$ behaves more linearly than PZT. Thus, it is highly practical and preferable to use $\mathrm{PVF}_{2}$ in a closed loop in order to perform feedback control on a PZT actuator.

\section{Linearity of PZT and $\mathrm{PVF}_{2}$ Actuators}

To induce large strain on the testbed, the structure system shown in Figs. 1a and 10 was driven into resonance by exciting the PZT actuator with the system resonant frequency. The resonant frequency of the one-dimensionalcantileverplate was identified by using a dynamic signal analyzer. ${ }^{20}$ The transfer function of the second testbed configuration is shown in Fig. 12, which clearly shows that the first resonant frequency of the testbed is around $13 \mathrm{~Hz}$.

Two types of driving sources, which are voltage and current sources, ${ }^{21}$ were used to excite the tested (Fig. 13) at $13 \mathrm{~Hz}$, and both $\mathrm{PVF}_{2}$ and AVID generated sensing signas for comparisons. The voltage source introduces prespecified voltage to PZT no matter what state PZT is in. On the other hand, the current source injects a prespecified amount of current to the PZT. Considering that the charge is the time integration of the current, driving PZT by using a current source is like driving PZT by specifying the externally applied charge. As was just mentioned, driving PZT by using the voltage source primarily utilizes the $\boldsymbol{e}$ constants of PZT, whereas driving PZT by the current source utilizes the $\boldsymbol{h}$ constants.

The voltage source used was a Wavetek Model 182A Function Generator, ${ }^{22}$ and the current source used was a Keithley Model 2420 $3 \mathrm{~A}$ Source Meter. ${ }^{23}$ To compare the hysteresis effects induced by

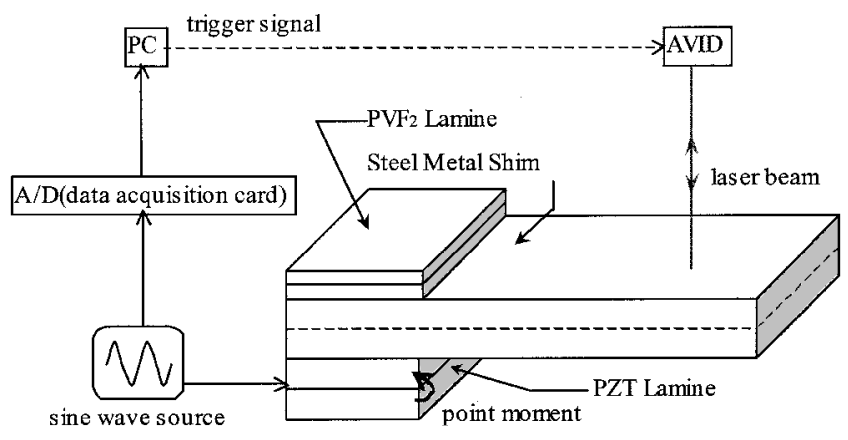

Fig.13 Experimental setup for examining driving source influence and the hysteresis effect of PZT.

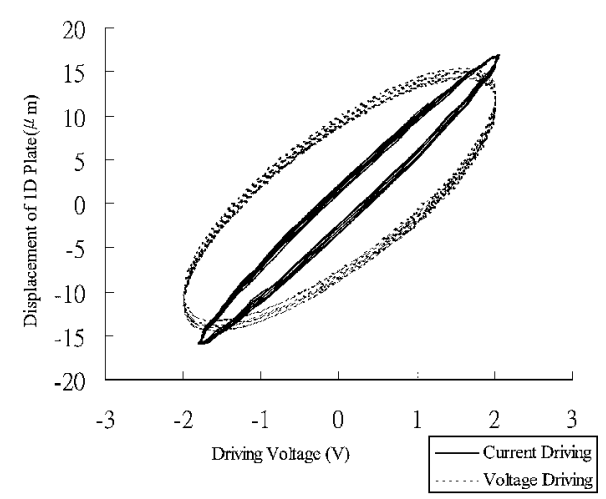

Fig. 14 Comparison of PZT linearity when driven by a voltage source and a current source.

these two different sources fairly, the equivalent externally applied voltage exerted by both sources was set between $-2 \mathrm{~V}$ to $2 \mathrm{~V}$. It is clear from the experimental data shown in Fig. 14 that driving PZT by using the current source instead of the voltage source creates a far more linear actuator. This result indicates that if linearity is of concern for a PZT actuator a current source driving should be considered.

\section{Conclusions}

Two testbed configurations based on distributed or point sensors and actuators were examined to show their validity as control to structure interaction platforms. The underlying principles, three built-in optical configurations, built-in mechanical and microscopic systems, etc., of AVID were all studied in detail. The features of AVID presented indicate that this system is quite suitable for evaluating high-performancestructural systems. Cross calibration of two types of distributed $\mathrm{PVF}_{2}$ sensors and the two measurement modes of AVID were shown to be in excellent agreement. More specifically, the linear and angular displacement/velocity measurement modes of AVID verified the performance of distributed $\mathrm{PVF}_{2}$ sensors in measuring bending angle and bending displacement at any point on the testbed. The experimental results showed tht $\mathrm{PVF}_{2}$ acts more linearly than PZT in sensing mode. In addition, the corresponding piezoelectric actuator that can exert a point concentrated moment was also verified. The influence of driving sources on the linearity of piezoelectric actuators was verified experimentally. The experimental data also obtained showed that driving PZT by using a current source instead of a voltage source reduces the hysteresis effect.

\section{Acknowledgments}

The authors gratefully acknowledge the support of the National Science Council of Taiwan, Republic of China, under Project Numbers NSC 84-2622-E-002-007, NSC 85-2622-E-002-017R, NSC 86-2212-E-002-040, and NSC 86-2622-E-002-023E. In addition, the funding support of AHEAD Optoelectronics, Inc., through an Industrial-AcademicCollaborative Project under Tjing-Ling Industrial Research Institute is also greatly appreciated. 


\section{References}

${ }^{1}$ Lee, C. K., and O’Sullivan, T. C., "Piezoelectric Strain Rate Gages," Journal of the Acoustical Society of America, Vol. 90, No. 2, 1991, pp. 945-953.

${ }^{2}$ Lee, C. K., Wu, G. Y., Teng, Thomas C. T., Wu, W. J., Lin, C. T., Hsiao, W. H., Shih, H. C., Wang, J. S., Lin, Sam S. C., Lin, Colin, C., Lee, C. F., and Lin, Y. C., "A High Performance Doppler Interferometer for Advanced Optical Storage Systems," Japanese Journal of Applied Physics, Vol. 38, Pt. 1, No. 3B, 1999, pp. 1730-1741.

${ }^{3}$ Lee, C. K., Wu, G. Y., Wu, W. J., Yeh, K. S., and Lee, C. F., "Interference Apparatus for Measuring Absolute and Differential Motions of Same or Different Testing Surface,” U.S. Patent No. 5,949,546, 7 Sept. 1999.

${ }^{4}$ Lee, C. K., "Piezoelectric Laminates for Torsional and Bending Modal Control: Theory and Experiment," Ph.D. Dissertation, Inst. of Theoretical and Applied Mechanics, Cornell Univ., Ithaca, NY, June 1987.

${ }^{5}$ Lee, C. K., "Piezoelectric Laminates: Theory and Experiments for Distributed Sensors and Actuators," Intelligent Structural Systems, Kluwer Academic, Norwell, MA, 1992, pp. 75-167.

${ }^{6}$ Lee, C. K., "Theory of Laminated Piezoelectric Plates for the Design of Distributed Sensors/Actuators, Part I: Governing Equations and Reciprocal Relationships," Journal of the Acoustical Society of America, Vol. 87, No. 3, 1990, pp. 1144-1158.

7"American National Standards Inst./Inst. of Electrical and Electronics Engineers, Standard on Piezoelectricity," Std. 176, Inst. of Electrical and Electronics Engineers, New York, 1987.

${ }^{8}$ Timoshenko, S., and Woinowsky-Krieger, S., Theory of Plates and Shells, McGraw-Hill, New York, 1959, pp. 79-104.

${ }^{9}$ Lee, C. K., and Moon, F. C., "Modal Sensors/Actuators," Journal of Applied Mechanics, Vol. 57, June 1990, pp. 434-441.

${ }^{10}$ Balas, M. J., "Feedback Control of Flexible Systems," Journal of Theory and Applications, Vol. 23, No. 4, 1978, pp. 673-679.

${ }^{11}$ Tsai, S. W., and Hahn, H. T., Introduction to Composite Materials, Technomic, Lancaster, PA, 1980.

12“AVID, Advanced Vibrometer/Interferometer Device," AHEAD Optoelectronics, Inc., No. 13, Taipei, Taiwan, ROC.

${ }^{13}$ Lee, C. K., and Wu, T. W., "Differential Laser Interferometer for Nanometer Displacement Measurement," AIAA Journal, Vol. 33, No. 9, 1995, pp. 1675-1680.

${ }^{14} 504$ E Dual Mode Amplifier User Manual, Kistler Instrument Corp., Amherst, New York.

${ }^{15}$ AM 501 Operational Amplifier Instruction Manual, Tektronix, Inc., Wilsonville, OR, April 1987.

${ }^{16}$ Martin, G. D., "On the Control of Flexible Mechanical Systems," Ph.D. Dissertation, Dept. of Aeronautics and Astronautics, Stanford Univ., Stanford, CA, 1978.

${ }^{17}$ Instruction ManualModel 2775A, Endevco Corp., San Juan Capistrano, CA, May 1988

${ }^{18}$ PCI-MIO E Series User Manual, National Instrument Corp., Austin, TX, Jan. 1997.

${ }^{19}$ LabVIEWUser's Manual, National Instrument Corp., Austin, TX, 1997.

${ }^{20}$ HP35665A Dynamic Signal Analyzer Operator's Reference, Hewlett Packard Corp., Everett, WA, May 1991.

${ }^{21}$ Horowitz, H., and Hill, W., The Art of Electronics, Cambridge Univ. Press, New York, 1980.

${ }^{22} 182$ A Function Generator User Manual, Wavetek Corp. San Digeo, CA, 1985.

${ }^{23}$ Model 2420 3A SourceMeter" User's Manual, Kiethley Corp., Solon, OH, April 1997.

A. M. Waas Associate Editor 